\title{
El COSTO DE COORDINAR: NÚMERO DE CANDIDATOS PRESIDENCIALES EN AMÉRICA LATINA 1993-2010*
}

\author{
The cost of coordinating: the number of presidential candidates in Latin \\ American elections, 1993-2010
}

\section{DIEGO LUJÁN}

Universidad de la República

\section{RESUMEN}

El número de candidatos que compite en una elección puede tener consecuencias políticas significativas. En América Latina, la competencia presidencial muestra un número de candidatos significativamente alto en muchos países, lo cual contradice las expectativas teóricas, por tratarse de una competencia uninominal. Este trabajo ofrece una explicación para este fenómeno, basada en los costos de coordinación que enfrentan los candidatos en mercados electorales inestables y poco programáticos. A nivel empírico, el argumento se pone a prueba a partir datos de elecciones presidenciales que tuvieron lugar en dieciocho países latinoamericanos entre 1993 y 2010. La evidencia permite sostener la hipótesis planteada, en la medida que mercados electorales inestables y poco programáticos, están asociados a un mayor número de candidatos en competencia.

Palabras clave: elecciones presidenciales, América Latina, coordinación electoral, fallos de coordinación, sistemas electorales

\begin{abstract}
The number of candidates competing in elections may have significant political consequences. In Latin America, presidential contests in many countries yield a large number of candidates, which contradicts theoretical expectations based on the fact that this is effectively a single-member competition. I provide an explanation based on coordination cost incurred by candidatess in unstable and low-programmatic electoral markets. Given this cost, we should expect entry decisions to exceed the theoretical upper limit. At the empirical level, the explanation is tested using statistical models of presidential election data in 18 Latin American countries between 1993 and 2010. I find evidence in support of the hypothesis that unstable and low-programmatic electoral markets are associated with a larger than expected number of presidential candidates.
\end{abstract}

Key words: presidential elections, Latin America, electoral coordination, coordination failure, electoral systems

\footnotetext{
Agradezco a Juan Andrés Moraes por sus valiosos comentarios y sugerencias a lo largo de la elaboración de este artículo. Asimismo, agradezco a varios colegas que comentaron versiones preliminares del manuscrito, entre los que destaco a Daniel Buquet, Daniel Chasquetti, Gabriel Katz y Rafael Piñeiro, además de dos evaluadores anónimos de RCP. Este artículo forma parte del proyecto de investigación "Explicando el Número de Candidatos Presidenciales en América Latina", financiado por la Comisión Sectorial de Investigación Científica de la Universidad de la República.
} 


\section{INTRODUCCIÓN}

Elnúmero de candidatos que compiten en una elección puede tener consecuencias políticas significativas. A medida que aumenta la oferta de candidatos, es más probable que un candidato minoritario resulte ganador. Por otra parte, cuando el número de candidatos en competencia es muy alto, la decisión de voto se hace más compleja, debido a las exigencias que el número de candidatos impone sobre el comportamiento estratégico de los votantes. En cambio, cuando hay pocos candidatos en competencia, el número de votos desperdiciados es bajo, y esto viabiliza el voto estratégico de modo compatible con el equilibrio duvergeriano. ${ }^{1}$ Por tanto, el número de candidatos es un aspecto clave en la lógica de la coordinación estratégica en el plano electoral (Cox 1997).

Desde el punto de vista empírico, desde la tercera ola de democratización en América Latina se ha producido un proceso de fragmentación de la oferta partidaria (y por consiguiente, de candidatos) que ha estado en la base de las explicaciones de diversos problemas que han enfrentado las democracias de la región (Mainwaring 1993; Jones 1995, 1999, 2004). Entre estos problemas, se pueden contar: la excesiva personalización de la competencia política y la irrupción de outsiders en la competencia electoral (Corrales 2008, 2009), el deterioro del vínculo entre la ciudadanía y los partidos políticos, problemas de identificabilidad y rendición de cuentas vertical (Mainwaring y Shugart 1997). Además, según señalan algunos estudios, a medida que aumenta el número de candidatos que compiten por la presidencia, el tamaño del contingente legislativo del Presidente tiende a decrecer (Shugart y Carey 1992; Mainwaring 1993; Jones 1995), complicando la gestión de gobierno.

América Latina es la región del mundo en la que más ha predominado el presidencialismo, por lo cual, en los países de esta región, la competencia política está estructurada en torno a la competencia por la presidencia (Jones 1995, 1999; Stokes 2009: 9). En consecuencia, la fragmentación política se manifiesta, especialmente, en ese nivel, permeando al resto del sistema político. La proliferación de candidatos presidenciales en algunos países latinoamericanos, ha sido explicada a menudo sin recurrir a una teoría explícita, sino a consideraciones ad hoc. El argumento que aquí se presenta sostiene que dicha proliferación obedece a que la coordinación de la entrada estratégica es costosa para los candidatos, debido a la existencia de mercados electorales inestables y poco programáticos, que dificultan la coordinación de la oferta.

El trabajo está estructurado de la siguiente forma. La primera sección presenta un repaso teórico de la literatura sobre coordinación electoral y sus consecuencias sobre la fragmentación de la competencia. El segundo apartado desarrolla el argumento teórico acerca del costo de la coordinación estratégica del lado de la

Un equilibrio duvergeriano es aquel en el que sólo M+1 candidatos reciben votos, siendo M el número de cargos en disputa. El término hace mención a la ley de Duverger (1954), y fue acuñado originalmente por Palfrey (1984) y luego retomado por otros autores como Cox (1997) y Myerson y Weber (1993). 
oferta, explicitando la forma en que funciona el mecanismo causal que une la inestabilidad y la escasa programaticidad de los mercados electorales, con los fallos de coordinación en la entrada de candidatos a la competencia electoral. En la tercera parte, se presentan los datos sobre el número de candidatos presidenciales en América Latina durante el período 1993-2010, junto a los modelos empleados para la contrastación empírica de las hipótesis propuestas. Finalmente, el último apartado resume las principales implicaciones teóricas esbozadas en las secciones precedentes, repasa los resultados y concluye.

\section{COORDINACIÓN ELECTORAL Y NÚMERO DE CANDIDATOS}

La literatura sobre sistemas electorales sostiene que las reglas determinan, en gran medida, el número de candidatos que compiten en una elección (Duverger 1954; Rae 1971; Taagepera y Shugart 1989; Sartori 1994; Cox 1997). A través de la coordinación de la entrada estratégica, las élites ajustan su comportamiento a las expectativas sobre lo que harán los votantes. Cuando existe información suficiente y las expectativas de los agentes son correctas, las élites serán capaces de prever acertadamente las consecuencias del voto estratégico, disuadiendo de este modo la entrada de candidatos excedentes. ${ }^{2}$ En consecuencia, la coordinación de la entrada estratégica por parte de las élites políticas es el principal mecanismo para ajustar la oferta a niveles de equilibrio.

Mientras, cuando la oferta no logra coordinarse adecuadamente $-\mathrm{y}$, por tanto, no se produce el efecto reductor-, los votantes tienen la posibilidad de corregir este fallo y, mediante la coordinación del voto estratégico, pueden reducir el número de candidatos relevantes mediante el abandono sistemático de las candidaturas más débiles. En este caso, el equilibrio duvergeriano se restablece, existiendo solo $\mathrm{M}+1$ candidatos recibiendo votos.

Para explicar los fallos de coordinación, la mayor parte de la literatura se enfoca en el voto estratégico y, por lo tanto, en el lado de la demanda. En general, las explicaciones se basan en las condiciones que imposibilitan la coordinación del voto, fundamentalmente debido a problemas de información, formación de expectativas y de tipo de preferencias de los votantes (Cox 1997; Benoit 2001; Tavits y Annus 2006; Myatt 2007; Crisp et al. 2012; Lago y Martínez 2012).

Algunos modelos toman en cuenta el lado de la oferta, pero se centran en la entrada estratégica. En general, estos parten del supuesto de que competir es costoso (Feddersen et al. 1990; Shvetzova 1995; Osborne y Silivinsky 1996; Weber 1997; Indridason 2008), por lo que el número de candidatos en equilibrio debería reflejar el balance entre dichos costos y el beneficio - ponderado por la probabilidad - de obtener el cargo. Reed $(1990,2003)$ se pregunta si el equilibrio duvergeriano es causado por el comportamiento de los votantes o 
de las élites, llegando a la conclusión de que son estas últimas las que, a través de retiros inducidos por aprendizaje, reducen la oferta al nivel de equilibrio. Sin embargo, para Reed (2003), los retiros no se producen más que por el efecto mecánico de las reglas, luego de un tiempo de aprendizaje. No hay diferenciación programática, ni cambios en las condiciones bajo las cuales compiten los candidatos. El argumento presentado en este trabajo coincide con el de Reed, en cuanto a que el equilibrio duvergeriano se debe, principalmente, a la adecuada coordinación del lado de la oferta, pero se diferencia en cuanto los retiros son inducidos no por aprendizaje, sino por racionalidad de los agentes que se enfrentan a mercados electorales que pueden ser más o menos inestables y más o menos programáticos.

Por otra parte, los enfoques de modelos espaciales han incorporado la noción de entrada estratégica, partiendo de la literatura sobre organización industrial (Shepsle y Cohen 1990). Estos asumen que la entrada es endógena, es decir, está en parte determinada por las condiciones en las cuales los agentes deben decidir su ingreso en la competencia. Además, en general, asumen que la (re) localización de los agentes en el espacio es costosa (Hay 1976; Prescott y Vischer 1977), lo cual introduce un elemento de tensión que modifica los equilibrios del juego. De acuerdo a Shepsle y Cohen (1990), si los costos de entrada son pequeños en relación al beneficio de ganar el cargo, entonces el modelo de Palfrey (1984) es compatible con la entrada de un tercer candidato, por lo cual la variable clave es la relación entre el costo de entrada y el beneficio esperado. Sin embargo, para los modelos de competencia espacial, el número de candidatos solo es relevante si modifica la ubicación de los agentes en el espacio político. En general, estos modelos no pretenden explicar cuántos candidatos deciden competir en una elección, sino dónde se ubicarán en un espacio n-dimensional.

Resumiendo, la literatura sobre coordinación electoral considera que los fallos de coordinación pueden explicarse, fundamentalmente, como consecuencia de factores que afectan la capacidad de los votantes de coordinar sus decisiones de voto. Desde ese punto de vista, la literatura ha privilegiado la explicación, basándose en el lado de la demanda. Sin embargo, como se observa en la Figura 1, la secuencia de la coordinación electoral se inicia con la coordinación de la oferta, a partir de las decisiones de entrada de los candidatos. En esta etapa puede surgir un número de candidatos próximo al número de equilibrio o bien uno mucho mayor, producto del costo de coordinación que enfrentan. Solo cuando los candidatos han decidido su entrada, tiene lugar la coordinación del lado de la demanda, a través de las decisiones de voto. Por esa razón, es necesario prestar atención al lado de la oferta, pues un fallo en la coordinación de la oferta es condición necesaria para un fallo en la coordinación de la demanda. ${ }^{3}$

Dicho de otro modo, no es posible que obtengan votos más candidatos que aquellos que están en competencia. Por tanto, si la oferta se conforma de acuerdo a la regla de M+1, no hay lugar para el voto estratégico. 
Figura 1. Secuencia temporal de la coordinación estratégica

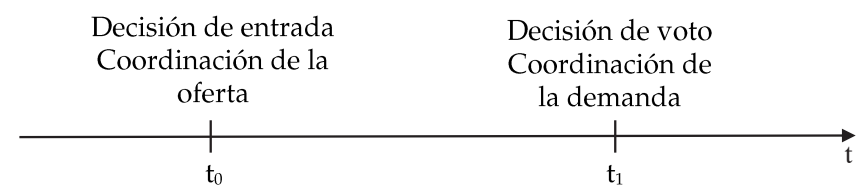

\section{EL COSTO DE COORDINAR: MERCADOS ELECTORALES INESTABLES Y POCO PROGRAMÁTICOS}

Este trabajo se centra en la primera etapa de la Figura 1. Por tanto, el argumento propuesto está basado en variables que afectan la entrada estratégica a partir de los costos que imponen sobre la coordinación entre potenciales candidatos. La inestabilidad y el grado de orientación programática del mercado electoral en el que compiten, generan mayores o menores costos de coordinación a los agentes al momento de decidir su entrada en la competencia. Estos costos inhiben transacciones que darían lugar a la conformación de una oferta eficiente (en el sentido de que reduce el número de competidores hasta el nivel de equilibrio $\mathrm{M}+1)$, redundando en un exceso de candidatos.

Partiendo de la literatura de transaction costs politics, ${ }^{4}$ podemos definir a la coordinación de la oferta como una transacción basada en el intercambio del retiro de un candidato a cambio de una compensación. Dicha compensación puede consistir en un beneficio programático (y, por tanto, puede medirse en policies), o en algún otro tipo de beneficio no programático (prestigio, cargos en el gobierno, futuras nominaciones, etcétera). La racionalidad de este intercambio radica en que - en un mercado electoral programático- el retiro de un candidato no es neutro para la competencia, sino que favorece al candidato más próximo ideológicamente. La coordinación de la entrada al nivel de las élites permite - mediante los retiros estratégicos- la conformación de un punto focal, lo cual facilita que muchos electores puedan votar de modo sincero, al sustraer varias opciones del menú electoral. En el extremo, si la conformación de la oferta es eficiente (es decir, se ajusta a la regla de $\mathrm{M}+1$ ), el voto estratégico no tiene lugar, pues los votantes se enfrentarán a un menú de opciones, tal que no quedan candidatos no viables a quienes abandonar.

La idea de que un retiro estratégico presenta las condiciones que podrían dar lugar a una transacción es plausible. Sin embargo, prácticamente no ha sido

Esta teoría considera que los agentes políticos interactúan en base a transacciones, es decir a intercambios de bienes políticos que están sujetos a costos de transacción (Weingast y Marshall 1988; North 1990; Dixit 1996; Epstein y O’Halloran 1999). 
considerada por la literatura. Cox (1997) sugiere la posibilidad de que un retiro estratégico pueda ser compensado, pero no desarrolla el tema. El autor señala:

[...] those elites who foresee that their own candidates or lists will bear the brunt of strategic desertion are likely to decide that mounting a (hopeless) campaign is not worth the cost, and seek instead to throw their support behind more viable candidates or lists (presumably for a price). To the extent that withdrawals of this sort do occur, the number of competitors will of course decrease (Cox 1997: 151, el resaltado es mío).

En el mismo volumen, sostiene: "It may be that contributors give only (or mostly) to frontrunning candidates, or that trailing candidates try to sell their endorsement to front-runners" (Cox 1997: 89, el resaltado es mío).

Para que la transacción recién caracterizada pueda tener lugar, es preciso que los mercados electorales presenten ciertas características que las faciliten o que, en otras palabras, presenten bajos costos de transacción. Los mercados electorales estables y programáticamente estructurados, ofrecen condiciones favorables para que estas transacciones tengan lugar, ajustando la oferta de candidatos a un número próximo a la regla de $\mathrm{M}+1$.

En primer lugar, es necesario que el mercado electoral esté programáticamente estructurado por dos razones. Por un lado, y como ha sugerido Fey (2007), aún sin voto estratégico (es decir, con votantes sinceros), si los candidatos están programáticamente orientados, pueden obtener un resultado más próximo a su política ideal, simplemente absteniéndose de participar en la elección. Como he señalado, este mecanismo, multiplicado por $n$ retiros estratégicos, presenta una lógica similar a la conformación de un punto focal, ${ }^{5}$ facilitando la coordinación de la entrada a múltiples candidatos de similares preferencias. Por otro lado, aun cuando un candidato tuviera motivaciones no programáticas (cargos, prestigio, poder), en un mercado electoral programático puede resultarle conveniente la estrategia de retirarse a tiempo a cambio de una compensación, en particular si su probabilidad de ganar la elección es muy baja. De este modo, el candidato podría obtener, bien una mayor chance de que se adopte una política de su preferencia, un cargo en el gobierno o una promesa de futuras candidaturas.

En segundo término, cuando las preferencias electorales son muy inestables, la transacción en que se basa la coordinación de la oferta se hace más complicada.

\footnotetext{
Son varios los autores que se refieren con el término "candidato focal" a aquel que, por diversos motivos, adquiere cierta visibilidad respecto de sus competidores. El término seguramente refiere a la idea elaborada por Schelling (1960), según la cual, en juegos con múltiples equilibrios de Nash, cualquier cosa que vuelva la atención de los jugadores hacia alguno de esos equilibrios aumenta la probabilidad de que sea alcanzado. De este modo, el atributo de focalidad vuelve particularmente interesante a uno de los múltiples equilibrios. Del mismo modo, la "focalidad" de un candidato es la que permite, muchas veces, resolver los dilemas de coordinación a los que se enfrentan tanto las élites partidarias como los votantes, cuando existen múltiples equilibrios. Según Cox (1997: 160) la "focalidad" es un dispositivo de coordinación valioso, siempre que permita resolver el dilema en cuestión, es decir, que no es un atributo permanente de este tipo de juegos, ni de los jugadores. El uso de la categoría "candidato focal" no es sistemático, pero puede encontrarse, por ejemplo, en Myerson y Weber (1993: 112); Cox (1997: 3); Lago (2004); Boix (2007: 520), entre otros.
} 
En mercados electorales inestables, la alternativa de competir solo se vuelve muy atractiva para los candidatos y, por tanto, son más probables los fallos de coordinación del lado de la oferta. La inestabilidad vuelve incierto el valor de retiro estratégico, pues su 'valor en votos' —al momento de tomar las decisiones de entrada y salida de la competencia- puede ser muy distinto a aquel que tenía al momento de la elección (Figura 1). Por tanto, los votos con que se beneficia el candidato focal de la coordinación pueden ser superiores o inferiores a los esperados cuando tuvo lugar la transacción.

\section{Implicaciones del argumento propuesto. Hipótesis}

La coordinación de la entrada estratégica puede modelarse como una transacción entre candidatos que aspiran a competir en una elección. Como en todo intercambio, es crucial medir adecuadamente el valor de lo que se intercambia ¿Cuál es el valor de la compensación por el retiro estratégico? En realidad, este dependerá de la proximidad entre la política preferida por el candidato que se retira y aquella preferida por el candidato focal beneficiado por dicho retiro. Otra vez, no es que a los candidatos solo les interesen las policies, sino que, al estar programáticamente estructurado, el mercado electoral responderá a los retiros del modo esperado, es decir beneficiando al candidato más próximo. De este modo, se facilita la transacción y el candidato sobreviviente recibe los votos que hubieran ido al que se retira, mientras este último recibe la compensación que — como se señaló en la sección anterior- puede ser simplemente programática (en tanto la política que aplicará el candidato beneficiado por su retiro será muy similar a la de su preferencia) o bien estar asociada a algún otro activo (cargos, futuras nominaciones, prestigio social, etcétera).

Esta medida nos permite estimar el beneficio en votos que espera recibir el candidato focal que continúa en carrera. Para que pueda medirse el intercambio, es necesario, entonces, que los votantes respondan sobre la base de posiciones definidas en el espacio político-ideológico. Estas deben ser más o menos estables y deben tener un significado sustantivo en materia de políticas públicas. En otras palabras, el mercado electoral debe estar estructurado en base a la competencia programática. Cuando esto sucede, los costos de transacción para la coordinación de la oferta electoral se reducen, dado que es posible valorar y compensar los retiros estratégicos. Lo que diferencia a los mercados electorales programáticos de los que no lo son es que los candidatos pueden calcular cuál es el valor de un retiro, pues los votos del candidato que se retira no se distribuirán aleatoriamente entre el resto de los candidatos en carrera, sino que beneficiarán al aspirante más próximo. Este mecanismo es independiente de las motivaciones de los políticos (prestigio, poder, dinero, cargos), pues está basado en la respuesta de los votantes ante el retiro de su candidato preferido y no en el objetivo de los candidatos. En los mercados no-programáticos, este mecanismo se ve fuertemente limitado, pues no es posible asegurar que los votantes del candidato que se retira se pasarán en conjunto a otro candidato en particular. 
Dado que el grado de diferenciación programática entre los agentes que componen el sistema se expresan en la polarización ideológica (Sani y Sartori 1983; Dalton 2008), es esperable que mayores niveles de esta a nivel sistémico reflejen mercados electorales más programáticos. Y viceversa: bajos niveles de polarización ideológica a nivel del sistema (y por ende de diferenciación programática) estarían expresando que la competencia política no se desarrolla en base a propuestas programáticas, sino en base a otro tipo de apelaciones o vínculos. De allí se deriva la primera hipótesis:

Hipótesis 1. A mayor nivel de polarización ideológica, menor número de candidatos en competencia.

Por otra parte, dado que el flujo de beneficios puede estar diferido en el tiempo - en particular para los candidatos que se retiran de la competencia-, la incertidumbre asociada al valor futuro del bien intercambiado es relevante. En especial, en ambientes inestables, el valor descontado a futuro de un retiro estratégico puede resultar tan bajo que vuelva inconveniente la transacción. Una excesiva inestabilidad en el ambiente, puede volver más conveniente entrar en la competencia a pesar de una baja probabilidad de éxito, pues siempre resulta tentador competir solo antes que desistir y favorecer a otro candidato, por más próximo que este se encuentre programáticamente. Por esa razón, si la coordinación es una transacción, la inestabilidad del ambiente en que compiten los actores puede impedir o limitar la coordinación de la entrada estratégica, al dificultar los compromisos intertemporales. ${ }^{6}$ En consecuencia, los mercados electorales más volátiles aumentan el costo de la coordinación de la oferta electoral. De allí, se deriva nuestra segunda hipótesis:

Hipótesis 2. A mayor nivel de volatilidad electoral, mayor número de candidatos en competencia.

La siguiente sección avanza en el testeo empírico de las dos hipótesis. Previamente, es muy importante hacer una aclaración. Como se ha destacado, existen numerosas variables que afectan la entrada de candidatos en una elección. Aquellas que surgen de la literatura disponible, serán incorporadas como variables de control. Sin embargo, también existen factores idiosincráticos que resulta imposible identificar o aislar para incorporar en el modelo. En consecuencia, más que proponer un modelo que explique definitivamente la entrada de candidatos, lo que se busca es testear las hipótesis propuestas, incorporando tanta información como sea posible.

\footnotetext{
Que la inestabilidad de la competencia electoral puede incidir sobre el número de candidatos en equilibrio no es algo enteramente novedoso. Grofman et al. (2009: 3) señalan que los equilibrios no duvergerianos pueden verse facilitados por la inestabilidad en las preferencias o en la formación de la oferta: "If reliable expectations about winners and losers are not available because party systems are in flux (...) then we can have non-Duvergerian outcomes" (el resaltado es mío).
} 


\section{NÚMERO DE CANDIDATOS PRESIDENCIALES EN AMÉRICA LATINA}

Este apartado presenta el análisis empírico de las dos hipótesis propuestas, a partir de datos de elecciones presidenciales en dieciocho países de América Latina, entre 1993 y 2010. ${ }^{7}$

La variable dependiente $(n c p)$ es el número de candidatos presidenciales que compitieron en cada elección. Pese a que la mayor parte de los test empíricos sobre esta temática utiliza como indicador el número efectivo de candidatos/ partidos, existen buenas razones para considerar que el índice de Laakso y Taagepera (1979) no es el mejor indicador en este caso. Como han notado Grofman et al. (2009), la utilización del número efectivo ha llevado a sobreestimar la pertinencia de la predicción del equilibrio duvergeriano. ${ }^{8}$ Cuando se observa al número de partidos que obtienen votos en lugar del número efectivo, la predicción se vuelve menos robusta. Lo mismo sucede cuando se miden candidatos que reciben votos en elecciones presidenciales.

Por otra parte, en este trabajo, lo que se pretende es medir la entrada estratégica o, en otras palabras, la fragmentación de la oferta. El número efectivo de candidatos no es una buena medida de la entrada estratégica, pues el indicador recoge los efectos del voto estratégico que solo puede ser reductor (ver Figura 1). Dado que el indicador creado por Laakso y Taagepera está afectado tanto por la oferta como por la demanda, no puede ser utilizado como un indicador de fragmentación de la oferta (Jones 1999: 175).

Por tanto, la forma más clara y directa de medir la oferta electoral es, simplemente, el número total de candidatos. Por supuesto, este indicador no permite ponderar las candidaturas por peso electoral, pero eso es en realidad una virtud del indicador más que una limitación. Si lo que se pretende es estudiar las decisiones de entrada de candidatos en una elección, entonces no hay un motivo a priori para descartar a ciertos candidatos que, al final del camino, recibieron pocos votos. La ponderación solo puede surgir a posteriori, cuando los votos ya fueron emitidos. Esto no es otra cosa que el número efectivo de candidatos, el número bruto ajustado por el tamaño electoral de la candidatura.

Utilizar como indicador el número de candidatos produce, sin embargo, un problema. No es posible deshacerse de los candidatos "testimoniales", es decir, de aquellos que corren la carrera presidencial buscando en realidad otros fines. Ya sea por motivos puramente personales o idiosincráticos, ciertos individuos

Los dieciocho países son Argentina, Bolivia, Brasil, Chile, Colombia, Colombia, Costa Rica, Ecuador, El Salvador, Guatemala, Honduras, México, Nicaragua, Panamá, Paraguay, Perú, República Dominicana, Uruguay y Venezuela.

8 "In Duverger's original formulation the "number" of political parties is simply ns, the number of parties whose representatives are elected. But virtually all of the tests of Duverger's law (and Duverger's hypothesis) use not ns, but rather the Laakso -Taagepera index of the effective electoral number of parties" (Grofman et al. 2009:6). 
podrían considerar la posibilidad de presentar su candidatura a la presidencia, especialmente bajo un sistema que presenta bajas barreras a la entrada de candidatos. Esta eventualidad no debería ser descartada a priori, aunque, tendencialmente, estos casos deberían ser marginales, pues ciertamente es muy costoso competir por la presidencia. Por otra parte, en numerosos países de la región, las candidaturas independientes no solo están fuertemente limitadas, sino que en muchas ocasiones son prohibidas por la ley. Una de las implicaciones del argumento teórico sostenido en este trabajo es que las candidaturas relevantes - o focales - se construyen a partir de la deserción de candidaturas a priori no viables, que son intercambiadas por una compensación. En la medida en que este proceso funciona cabalmente, es esperable que las candidaturas marginales salgan de la competencia en favor de las focales, que, por definición, son pocas. Que esto suceda efectivamente o no es una cuestión empírica, que deberá resultar del examen de los datos, tarea de la que se ocupa el presente acápite. En consecuencia, no hay razones teóricas para descartar a priori a los candidatos marginales, toda vez que su existencia forma parte central del argumento teórico aquí sostenido. De todos modos, para tomar en cuenta este fenómeno, se incorporan a los modelos empíricos algunas variables de control que son discutidas más adelante.

Tabla 1. Número de Candidatos Presidenciales en América Latina 1993-2010

\begin{tabular}{|c|c|c|c|c|c|c|}
\hline País & Elecciones & Años & Media & Des. Std. & Min. & Max. \\
\hline Argentina & 4 & $1995,1999,2003,2007$ & 14 & 3,26 & 10 & 18 \\
\hline Bolivia & 5 & 1993, 1997, 2002, 2005, 2009 & 9,8 & 1,79 & 8 & 12 \\
\hline Brasil & 2 & 2006,2010 & 8 & 1,41 & 7 & 9 \\
\hline Chile & 4 & $1993,1999,2005,2009$ & 5 & 1,15 & 4 & 6 \\
\hline Colombia & 5 & $1994,1998,2002,2006,2010$ & 11,6 & 4,22 & 7 & 18 \\
\hline Costa Rica & 5 & 1994, 1998, 2002, 2006, 2010 & 11 & 2,91 & 7 & 14 \\
\hline Ecuador & 5 & 1996, 1998, 2002, 2006, 2009 & 9,4 & 2,70 & 6 & 13 \\
\hline El Salvador & 4 & $1994,1999,2004,2009$ & 5 & 2,45 & 2 & 7 \\
\hline Guatemala & 4 & $1995,1999,2003,2007$ & 13,75 & 3,77 & 11 & 19 \\
\hline Honduras & 5 & 1993, 1997, 2001, 2005, 2009 & 4,8 & 0,45 & 4 & 5 \\
\hline México & 3 & $1994,2000,2006$ & 6,67 & 2,08 & 5 & 9 \\
\hline Nicaragua & 3 & $1996,2001,2006$ & 10 & 10,44 & 3 & 22 \\
\hline Panamá & 4 & 1994, 1999, 2004, 2009 & 4,25 & 1,89 & 3 & 7 \\
\hline Paraguay & 3 & $1993,1998,2003$ & 7,33 & 2,89 & 4 & 9 \\
\hline Perú & 3 & $1995,2001,2006$ & 14 & 6 & 8 & 20 \\
\hline $\begin{array}{l}\text { República Do- } \\
\text { minicana }\end{array}$ & 5 & 1994, 1996, 2000, 2004, 2008 & 12,6 & 4,72 & 7 & 20 \\
\hline Uruguay & 4 & $1994,1999,2004,2009$ & 8,25 & 4,72 & 5 & 15 \\
\hline Venezuela & 2 & 1998,2000 & 7 & 5,66 & 3 & 11 \\
\hline Total & $(\mathrm{N}=70)$ & & 9,16 & 4,65 & 2 & 22 \\
\hline
\end{tabular}

Fuente: elaboración propia 
El número de candidatos presidenciales ha presentado una considerable variación entre y dentro de los países de América Latina durante el período considerado. Como puede observarse en la Tabla 1, los países que han presentado mayor cantidad de candidatos presidenciales en promedio, son: Perú, Argentina, Guatemala y República Dominicana, con un promedio superior a los doce candidatos. En el extremo opuesto: Panamá, Honduras, Chile y El Salvador no han superado los cinco candidatos presidenciales en promedio.

\section{Midiendo las variables independientes}

La primera variable independiente considerada en el modelo es la polarización ideológica (polarización), medida según del índice de Taylor y Herman (1971), ${ }^{9}$ a partir de encuestas a legisladores realizadas por la Encuesta de Élites de la Universidad de Salamanca. ${ }^{10}$ El nivel de polarización es un rasgo sistémico que mide la distancia que existe entre los distintos agentes que componen el sistema, ponderada por su peso electoral. ${ }^{11}$ Dado que la distancia refiere a las ubicaciones de dichos agentes en el continuo ideológico, la misma expresa distintas preferencias de policies, que en general deberían guardar cierta coherencia. Por lo tanto, un sistema político con niveles altos de polarización ideológica estaría expresando diferencias programáticas profundas entre los agentes que compiten dentro del mismo.

La segunda variable independiente es la volatilidad electoral (volatilidad), calculada según el índice de Pedersen (1979). ${ }^{12}$ La volatilidad relaciona las preferencias de los votantes entre dos elecciones sucesivas, por lo cual es una medición que está afectada por los resultados de la elección en curso,

$9 \quad$ El índice se calcula del siguiente modo: $P=\sum_{i=1}^{n} v_{i}\left(x_{i}-\bar{x}\right)^{2}$, donde $v_{i}$ es el porcentaje de votos obtenido por el partido iésimo; $x_{i}$ es la ubicación de dicho partido en el eje izquierda-derecha y es la media ideológica del sistema. Los resultados que se presentarán a continuación no son sustantivamente distintos cuando se utilizan otros indicadores de la polarización ideológica como el utilizado por Huber (1989) o por Dalton (2008).

$10 \quad$ A los legisladores se les pregunta: "Como recordará, cuando se habla de política se utilizan normalmente las expresiones izquierda y derecha. En esta tarjeta hay una serie de casillas que van de izquierda a derecha ¿dónde ubicaría Ud. a su propio partido?".

${ }_{11}$ Los partidos que, por su tamaño electoral no acceden al legislativo, no afectan el valor del índice. En realidad, la polarización ideológica es un valor sistémico, no es un atributo de ningún actor individual. Por otra parte, el índice de Taylor y Herman es un indicador de polarización ideológica ponderado por tamaño electoral, lo que significa que partidos muy pequeños — aun cuando fueran muy extremistas_-incidirán solo marginalmente en el valor sistémico. En realidad, lo que importa para caracterizar a un sistema según su nivel de polarización es la distancia ideológica entre los partidos relevantes que lo componen.

12 El índice de Pedersen se calcula del siguiente modo: $\mathrm{V}=\frac{1}{2} \sum_{i=1}^{n}\left|p_{i}(t+1)-p_{i}(t)\right|$, donde $p_{i}(t+1)$ es la proporción de votos obtenida por el partido iésimo en una elección, y $p_{i}(t)$ es la proporción de votos obtenida por el partido iésimo en la elección anterior. Para calcular la volatilidad se han utilizado datos electorales de Nohlen (2005a y 2005b) y la Political Database of the Americas (s.f.) de la Universidad de Georgetown. Siempre que ha sido posible, se han calculado en base a las votaciones de los partidos para la cámara baja, debido a que la mayor parte de los estudios sobre la materia consideran este nivel para calcular la volatilidad, con lo cual se gana en capacidad de comparación. Por otra parte, las alternativas presentaban distintos problemas para su cálculo. Por un lado, las cámaras altas no siempre existen y, durante el período considerado, su integración no siempre respondió a la elección popular directa. Por otro, las elecciones presidenciales no son un buen indicador para medir la volatilidad a nivel comparado, debido a que distintos partidos compiten bajo diferentes etiquetas para la elección presidencial, pues las reglas lo permiten e incentivan en distintos países. 
pero también de la anterior. En cierta forma, la volatilidad permite reflejar el nivel de estabilidad y de certidumbre que enfrentan los agentes que compiten electoralmente en el momento en el que deben tomar sus decisiones de entrada. Si en una determinada elección un candidato recibió una cantidad de votos muy diferente de la que espera obtener en la siguiente, entonces el nivel de incertidumbre que enfrenta es alto. De lo contrario, si el porcentaje de votos que cierto candidato obtuvo en la elección anterior es similar al que espera obtener en la siguiente, entonces el mercado electoral es estable, y esto permite que existan transacciones como las que conforman la coordinación de la oferta. Obviamente, la estabilidad no tiene por qué ser un atributo permanente de los mercados electorales. Sin embargo, aunque existan fluctuaciones en los porcentajes de votos durante un cierto período, en la medida en que los cambios esperados sean previsibles, esto no afecta sustantivamente el comportamiento estratégico de los agentes.

Por otra parte, se incorporan al modelo variables de control derivadas de la revisión de la literatura. El primer factor a tener en cuenta es el sistema electoral. Como sabemos, el tipo de sistema electoral utilizado para una elección tiene incidencia sobre el número de candidatos en competencia (Duverger 1954; Rae 1971; Taagepera y Shugart 1989; Sartori 1994; Cox 1997). Por ese motivo, se añade la variable (regla) que mide cuan restrictivo es el sistema electoral utilizado en las elecciones presidenciales comprendidas en este trabajo. En América Latina, existen tres fórmulas para la elección presidencial: la variable regla toma el valor 1 en el caso de la mayoría relativa, 2 en el caso de la mayoría calificada, también llamada de umbral reducido, y 3 en el caso de la mayoría absoluta. Los dos últimos tipos de regla requieren la posibilidad de que exista una segunda vuelta entre los candidatos más votados. Se espera que los sistemas más restrictivos estén relacionados con un menor número de candidatos presidenciales, en la medida en que disuaden la entrada de terceros candidatos. ${ }^{13}$ Por tanto, el número de candidatos debería crecer en la medida que se pasa de un sistema de mayoría relativa a uno de mayoría calificada, por ejemplo.

Asimismo, la entrada de candidatos depende de cuan exigentes sean las barreras para presentar y sostener una candidatura. Si están permitidas las candidaturas independientes, entonces las barreras a la entrada son más bajas que si estuvieran prohibidas. Cuando un individuo puede presentar su candidatura sin necesidad de contar con un aval partidario, se simplifica considerablemente el proceso de nominación, y la entrada en la competencia se hace más fácil. Por otra parte, existen barreras de distinto tipo para formar un nuevo partido en aquellos países en los cuales es necesario un aval partidario para competir por la presidencia. Del mismo modo, el financiamiento de los partidos puede ser público o provenir de su propia capacidad para recaudar contribuciones y aportes económicos. Si la financiación es, al menos en parte pública, es más

13 Sobre los motivos por los cuales los sistemas de doble vuelta aumentan los incentivos para la entrada de candidatos, puede verse Jones (2004:79). 
fácil impulsar una candidatura, pues se reduce el esfuerzo que deben hacer los nuevos partidos para acceder al mercado electoral. Lo mismo sucede con la base sobre la cual se calcula el aporte que el Estado realiza a los partidos. Si el Estado contribuye sobre la base de los resultados electorales pasados, es más difícil impulsar candidaturas nuevas que si el Estado distribuye recursos sobre una base de cálculo independiente de rendimientos electorales pasados. Resulta complicado mapear la gran heterogeneidad existente en América Latina sobre la base de estos cuatro criterios, pese a lo cual la variable (candidatura) pretende dar cuenta de estos factores a través de un índice ordinal que varía entre 0 (cuando es más difícil lanzar una candidatura presidencial, pues existen altas barreras a la entrada) y 4 (cuando es más fácil lanzar candidaturas presidenciales). ${ }^{14}$

Por otra parte, si un Presidente en ejercicio busca la reelección, es menos probable el ingreso de candidatos desafiantes (Jones 1999) y, en consecuencia, es esperable una menor entrada de candidatos. Para ello, se añade la variable de control (reelección), que asume el valor 1 cuando un Presidente en ejercicio busca su reelección y el valor 0 en caso contrario.

La relación entre la fragmentación a nivel legislativo y el número de candidatos presidenciales puede tener dos caras. Por una parte, cuando la presidencia es un "gran premio", esto puede incentivar la conformación de pocos partidos legislativos que se organicen en torno a la disputa presidencial (Shugart y Carey 1992; Jones 1995; Cox 1997; Mainwaring y Shugart 1997; Golder 2006). Sin embargo, también es posible que sea la fragmentación legislativa la que incida sobre la competencia presidencial, como razonablemente ha argumentado Jones (1999). Algunos partidos que no tienen chances de ganar la elección presidencial aún pueden considerar conveniente presentar candidatos presidenciales, solo para poder competir por escaños legislativos, especialmente en elecciones concurrentes bajo un voto de tipo conjunto, en el cual no es posible para el votante separar el orden legislativo del ejecutivo. En las setenta elecciones consideradas en este trabajo, nueve de cada diez fueron concurrentes, razón por la cual cabría esperar que la competencia presidencial pueda ser afectada por la fragmentación a nivel legislativo. Por ese motivo, se incorpora una variable de control que mide el número efectivo de partidos legislativos (NEPL).

La organización de carácter federal de algunos países latinoamericanos podría incidir sobre el número de candidaturas presidenciales (Johnston y Cutler 2009: 92), en la medida en que los incentivos para la aparición de nuevos partidos a nivel subnacional podrían diferir de aquellos que existen a nivel nacional. En consecuencia, partidos regionales o provinciales podrían encontrar conveniente promover candidatos presidenciales aún sin tener reales posibilidades de ganar

14 El índice se calcula sumando el puntaje de cada una de las cuatro dimensiones mencionadas: candidatos independientes, requisitos para crear y sostener un partido político, financiamiento público y base de cálculo del financiamiento. Por ello, la variable oscila entre 0 y 4 . Existen, desde luego, otros factores que podrían agregarse al índice. Sin embargo, la dificultad para conseguir los datos conspira en parte contra este ideal, así como la dificultad para rastrear los cambios ocurridos dentro de cada uno de los dieciocho países analizados. Los datos utilizados corresponden a ACE Project: The Electoral Knowledge Network (s.f.). 
la elección. Por lo tanto, se esperaría que los estados más federales tuvieran un mayor número de candidatos presidenciales que los estados con una organización no federal. Para ello, se agrega la variable de control (federalismo) que mide el nivel de federalismo en una escala que va del 1 al 5, donde 1 es un nivel máximo de federalismo y 5 es un nivel máximo de unitarismo. ${ }^{15}$

Tabla 2. Estadísticos descriptivos de las variables de control

\begin{tabular}{lcccc}
\hline \multicolumn{1}{c}{ Variable } & Media & Desviación estándar & Min. & Max. \\
\hline Regla & 2,18 & 0,84 & 1 & 3 \\
Candidatura & 2,53 & 1,07 & 1 & 4 \\
Federalismo & 3,57 & 1,5 & 1 & 5 \\
Reelección & 0,13 & 0,34 & 0 & 1 \\
\hline NEPL & 3,85 & 1,74 & 1,85 & 10,37 \\
\hline
\end{tabular}

\section{Análisis de los datos y discusión de los resultados}

La Tabla 3 presenta los resultados de tres modelos diferentes de regresión binomial negativa truncada en cero (ver Hilbe 2011). El Modelo 1 tiene como predictores únicamente las dos variables independientes propuestas en las Hipótesis 1 y 2, además del control regla. ${ }^{16}$ Como puede observarse, las dos variables independientes consideradas tienen impactos significativos y presentan un signo consistente con el efecto esperado. A mayor nivel de polarización ideológica está asociado un menor número de candidatos presidenciales, mientras que a mayor nivel de volatilidad electoral se relaciona un mayor número de candidatos. Adicionalmente, la regla de elección presenta un efecto positivo pero no significativo. En consecuencia, ambas hipótesis reciben respaldo empírico al encontrarse evidencia sobre el impacto de la polarización ideológica y la volatilidad electoral sobre el número de candidatos que compiten por la presidencia en América Latina. ${ }^{17}$

El Modelo 2 utiliza el mismo método de estimación con el agregado del resto de las variables de control detalladas. Como puede observarse, las dos variables independientes mantienen impactos significativos y con los signos esperados

15 Los datos son los utilizados por Gerring y Thacker (2004).

16 Dado que el número de candidatos en competencia podría estar afectado por la heterogeneidad social (Amorim Neto y Cox 1997), los mismos modelos fueron estimados con un control adicional que mide el nivel de heterogeneidad étnica (datos utilizados por Alesina et al 2003). Esta variable no reportó significación estadística en ninguno de los tres modelos, razón por la cual no es incluida en la Tabla 3.

17 Cuando los mismos tres modelos fueron corridos con la variable dependiente modificada por un umbral del $1 \%$ (con la finalidad de filtrar los candidatos "testimoniales"), los resultados no difieren demasiado de los presentados en la Tabla 2. La principal diferencia es que la variable polarización pasa a tener un $p$-value de 0,104 en el Modelo 1 . 
en las hipótesis. En este caso, la variable regla adquiere significación estadística y con el signo esperado.

Finalmente, el Modelo 3 es idéntico al 2, pero con el agregado de variables binarias por país, para controlar por el efecto de cada unidad dentro del panel. Como puede observarse, los resultados no difieren demasiado de los reportados en los dos modelos anteriores. Las dos variables independientes mantienen significación estadística, así como el signo esperado, mientras que el resto de las variables no son significativas.

Tabla 3. Efectos del costo de coordinar sobre el número de candidatos presidenciales en América Latina

\begin{tabular}{lrrr}
\hline \multicolumn{1}{c}{ Var. Dep. (ncp) } & Modelo 1 & Modelo 2 & \multicolumn{1}{c}{ Modelo 3 } \\
\hline \multirow{2}{*}{ Volatilidad } & $0,010^{* * *}$ & $0,011^{* * *}$ & $0,014^{*}$ \\
& $(0,003)$ & $(0,003)$ & $(0,005)$ \\
Polarización & $-0,101^{* * *}$ & $-0,104^{* * *}$ & $-0,104^{* * *}$ \\
& $(0,03)$ & $(0,027)$ & $(0,027)$ \\
Regla & 0,132 & $0,176^{*}$ & $-0,177$ \\
& $(0,071)$ & $(0,074)$ & $(0,119)$ \\
Candidatura & & $-0,057$ & 0,208 \\
& & $(0,052)$ & $(0,554)$ \\
Federalismo & & $-0,043$ & 0,110 \\
& & $(0,038)$ & $(0,106)$ \\
Reelección & & 0,123 & $-0,059$ \\
& & $(0,160)$ & $(0,125)$ \\
NEPL & & $-0,058$ & $-0,053$ \\
Constante & 1,74 & $(0,030)$ & $(0,045)$ \\
Dummies por país & No & 2,12 & 2,32 \\
$N^{\circ}$ casos & 66 & No & Si \\
\hline
\end{tabular}

Nota: errores estándar entre paréntesis

* Significativo al 0,05

*** Significativo al 0,001

Por otra parte, para poder ilustrar el impacto que cada una de las variables independientes propuestas en las Hipótesis 1 y 2 tiene sobre el número de candidatos, se procedió a realizar una simulación ${ }^{18}$ de distintos escenarios que pueden resultar de interés. Con esa idea, se simularon dos escenarios con países

18 Las simulaciones se llevaron a cabo mediante los paquetes Clarify para STATA (ver King et al. 2000) y Zelig para R (ver Imai et al. 2006). 
idénticos en todas las variables consideradas en el modelo, excepto la volatilidad electoral y la polarización ideológica. El valor de cada una de las variables se fijó en la moda, es decir su valor más repetido para el caso de variables ordinales o binarias o en su media (cuando la misma tiene sentido teórico y existe), de modo de simular el país más representativo posible de los que conforman la muestra. Se trata de un país con una organización administrativa unitaria (federal $=5$ ), con regla electoral de mayoría calificada (regla=2), con un nivel intermedio de dificultad para presentar una candidatura presidencial (candidatura $=3$ ) y sin un Presidente buscando su reelección inmediata (reelección $=0$ ).

En el Escenario 1, se simuló un mercado electoral programático y estable y, por tanto, alta polarización ideológica y baja volatilidad electoral. Inversamente, en el Escenario 2 se simuló un mercado electoral inestable y poco programático, es decir con alta volatilidad electoral y bajo nivel de polarización ideológica. Los valores tanto de polarización ideológica como de volatilidad electoral fueron tomados de los promedios de los países con mayor y menor valor en ambas variables, de modo que el valor imputado refleje una situación empíricamente existente. ${ }^{19}$

El promedio de candidatos presidenciales bajo el Escenario 1 es de 3,35, mientras que en el Escenario 2 asciende a 13,57 de acuerdo a los resultados de la simulación. Esta considerable diferencia entre escenarios contribuye a reflejar el impacto sustantivo que - tanto la polarización ideológica como la volatilidad electoral- tienen sobre la coordinación estratégica de la oferta y, por tanto, en el número de candidatos que compiten en la elección. Un aspecto a resaltar es que, bajo el Escenario 1, el nivel de fragmentación promedio se sitúa muy próximo a un nivel de equilibrio duvergeriano, teniendo en cuenta la regla de mayoría calificada. En este caso, la regla de $\mathrm{M}+1$ haría esperar un número de candidatos igual a 3 , muy cercano al número promedio $(3,35)$. Esto significa que, bajo las condiciones del Escenario 1, el nivel de voto estratégico no necesita ser muy alto para llevar esa oferta de candidatos al nivel de equilibrio duvergeriano.

19 Para el Escenario 1 se tomó el promedio de polarización ideológica de El Salvador y el promedio de volatilidad electoral de Chile. Mientras, para el Escenario 2 se tomó el promedio de polarización ideológica de Paraguay y la volatilidad electoral promedio de Perú. 
Gráfico 1. Número esperado de candidatos presidenciales según escenarios 1 y 2

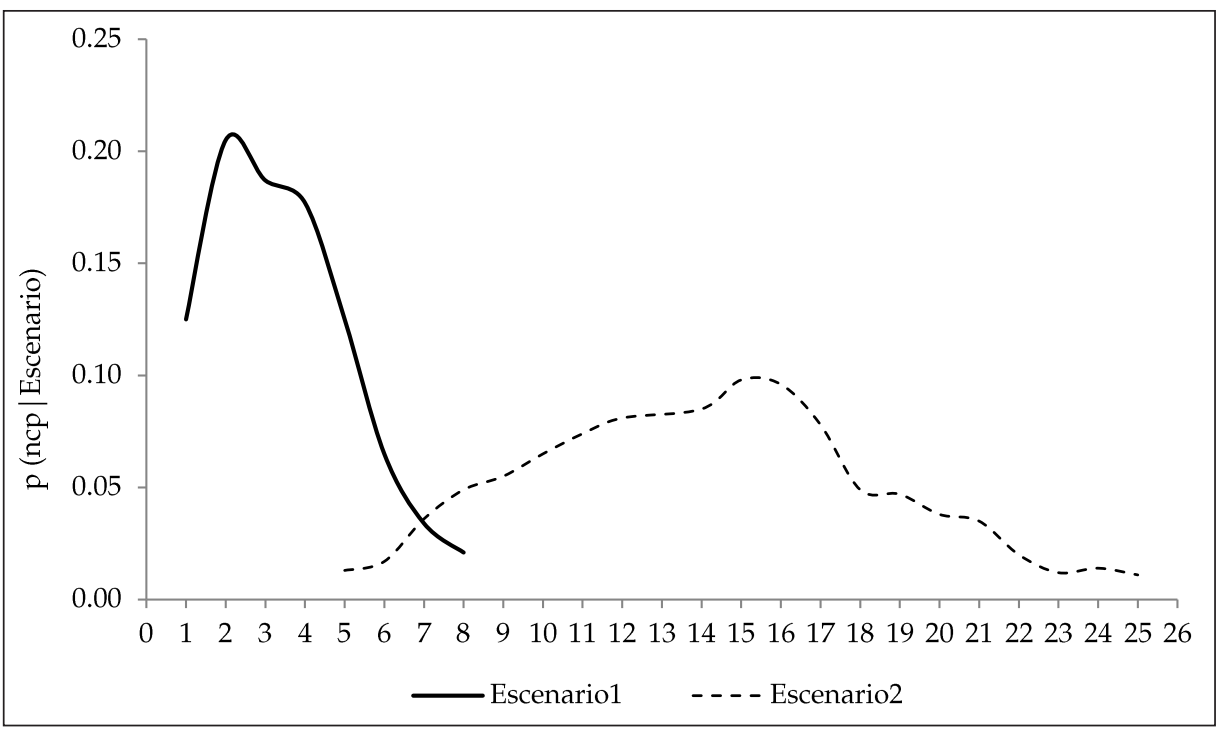

Finalmente, el análisis de primeras diferencias, esto es, la diferencia entre los valores esperados bajo ambos escenarios es, en promedio de 10,22 candidatos. Esto significa que, para cada valor que puede asumir la variable dependiente, existe entre ambos escenarios una diferencia de más de diez candidatos en promedio. Dado que lo único que cambia de un escenario a otro es el valor de polarización ideológica y de volatilidad electoral, debemos atribuir esta diferencia al efecto conjunto de ambas variables.

\section{CONCLUSIONES}

La coordinación electoral es el principal mecanismo que permite equilibrar el número de candidatos en función de la regla de elección. La literatura que explica los fallos de coordinación se ha centrado, mayormente, en el lado de la demanda, y ha descuidado aquellos del lado de la oferta. Una de las principales implicaciones del argumento que se ha presentado es que es necesario atender a estos últimos, pues son condición necesaria (aunque no suficiente) para un equilibrio no duvergeriano. En la conformación ineficiente de la oferta se inicia la secuencia que puede originar un fallo de coordinación, cuando el voto estratégico no alcanza a reducir el número de candidatos hasta el valor de equilibrio.

Como se ha descrito, no todas las condiciones de competencia son igualmente favorables para la coordinación del lado de la oferta. Desde la irrupción de las democracias de la tercera ola en América Latina, en la mayor parte de las elecciones presidenciales los partidos han sido incapaces de coordinar eficientemente la oferta de candidatos. En muchos casos, estos fallos en la 
coordinación de la oferta pudieron ser resueltos por la coordinación de la demanda. Los votantes han concentrado sus votos en unos pocos candidatos, reduciendo de ese modo el número de competidores relevantes.

Sin embargo, dados los exigentes requisitos informacionales, de experiencia previa y conocimiento adecuado de las consecuencias de las reglas electorales, la coordinación del voto estratégico no siempre es posible en los niveles que se necesitarían para restituir el equilibrio. Cuando el número de candidatos es exageradamente alto, se torna difícil para los votantes coordinar sus decisiones de voto, entre otras cosas porque no siempre resulta evidente a qué candidatos hay que abandonar y a cuáles apoyar en su lugar. En estos casos, el resultado es un número excesivo de candidatos que reciben votos.

En este trabajo se ha ofrecido una explicación para los fallos en la coordinación de la entrada estratégica a partir de los costos de transacción que enfrentan los candidatos que deben coordinar su entrada en la competencia. Para ello, se ha definido a la coordinación de la oferta electoral como una forma específica de transacción política en la cual un candidato intercambia su retiro a cambio de una compensación. Las condiciones para la transacción aparecen debido a que el retiro estratégico de un aspirante beneficia al sobreviviente más próximo programáticamente. A cambio de su retiro, el candidato que desiste de competir obtiene una compensación bajo la forma de un beneficio que puede ser programático (en virtud del cual la política que se aplicará estará más cerca de su propia preferencia) o no (cargos, futuras nominaciones, prestigio social, etcétera). En última instancia, lo relevante no es la motivación del candidato que se retira, sino cuál es la respuesta más probable de los votantes ante su retiro, pues eso es lo que diferencia a un mercado de tipo programático de uno que no lo es.

En mercados electorales inestables y poco programáticos, los costos de transacción inhiben la coordinación de la entrada estratégica mediante dos mecanismos. Por un lado, la inestabilidad de la competencia electoral vuelve muy difícil llegar a acuerdos temporalmente consistentes. En efecto, la constante aparición y desaparición de actores políticos, que en una elección tienen un muy buen desempeño pero desaparecen en la siguiente, puede volver inviable la coordinación entre candidatos. Los comportamientos intertemporales son incompatibles con ambientes cargados de incertidumbre e inestabilidad, pues "la sombra del futuro" pierde su capacidad de constreñir el comportamiento de los agentes. En contextos como estos, es imposible establecer con claridad cuáles candidatos son viables y cuáles no lo son, lo que aumenta la tentación de "competir solo", impidiendo las transacciones que dan lugar a la coordinación de la entrada estratégica. Por otro lado, en ambientes que propician una competencia política no programática, es difícil para los actores involucrados estimar el valor de lo que se intercambia en una transacción. 
Cuando ambas condiciones se encuentran presentes, el mercado electoral presenta un alto costo de coordinación, lo que permite explicar por qué hay países con un número tan elevado de candidatos presidenciales.

\section{REFERENCIAS}

ACE Project: The Electoral Knowledge Network. S.f. Recuperado en https:/ / www.aceproject.org. Alesina, Alberto, Arnaud Devleeschauwer, William Easterly, Sergio Kurlat y Romain Wacziarg. 2003. "Fractionalization". Journal of Economic Growth, 8(2): 155-194.

Amorim Neto, Octavio y Gary Cox. 1997. "Electoral Institutions, Cleavage Structures, and the Number of Parties". American Journal of Political Science 41(1): 149-174.

Benoit, Kenneth. 2001. "Two Steps Forward, One Step Back: Electoral Coordination in the Hungarian Elections of 1988". Ponencia presentada en la 2000 Annual Meeting of the American Political Science Association, Washington, D.C., August 31-September 3.

Boix, Carles. 2007. "The Emergence of Parties and Party Systems". En The Oxford Handbook of Comparative Politics, editado por Carles Boix y Susan Stokes. New York: Oxford University Press, 499-521.

Corrales, Javier. 2008. “Latin America's Neocaudillismo: Ex-Presidents and Newcomers Running for President... and Winning". Latin American Politics and Society 50(3): 1-35.

Corrales, Javier. 2009. "Volatilidad económica, debilidad de partidos y el neocaudillismo en América Latina". Journal of Democracy en Español 1: 55-76.

Cox, Gary. 1997. Making Votes Count: Strategic Coordination in the World's Electoral Systems. Cambridge: Cambridge University Press.

Crisp, Brian, Santiago Olivella y Joshua Potter. 2012. "Electoral Contexts that Impede Voter Coordination". Electoral Studies 31(1): 143-158.

Dalton, Russell 2008. "The Quantity and the Quality of Party Systems: Party System Polarization, Its Measurement, and Its Consequences". Comparative Political Studies 41(7): 899-920.

Dixit, Avinash. 1996. The Making of Economic Policy: A Transaction-Cost Politics Perspective. Cambridge: The MIT Press.

Duverger, Maurice. 1954. Political Parties: Their Organization and Activity in the Modern State. Methuen: Wiley.

Epstein, David y Sharyn O'Halloran. 1999. Delegating Powers. A Transaction Cost Politics Approach to Policy Making Under Aeparate Powers. Cambridge: Cambridge University Press.

Feddersen, Timothy, Itai Sened y Stephen Wright. 1990. "Rational Voting and Candidate Entry Under Plurality Rule". American Journal of Political Science 34(4): 1005-1016.

Fey, Mark. 2007. “Duverger's Law Without Strategic Voting". Working Paper, Department of Political Science. Rochester: University of Rochester.

Gerring, John y Strom Thacker. 2004. "Political Institutions and Corruption: The Role of Unitarism and Parliamentarism". British Journal of Political Science 34: 295-330.

Golder, Matt. 2006. "Presidential Coattails and Legislative Fragmentation". American Journal of Political Science 50(1): 34-48.

Grofman, Bernard, Shawn Bowler y André Blais. 2009. "Introduction: Evidence for Duverger's Law from Four Countries". En Duverger's Law of Plurality Voting: The Logic of Party Competition in Canada, India, the United Kingdom and the United States, editado por Bernard Grofman, André Blais y Shaun Bowler. New York: Springer, 1-11.

Hay, Donald 1976. "Sequential Entry and Entry-Deterring Strategies in Spatial Competition". Oxford Economic Papers New Series 28(20): 240-257.

Hilbe, Joseph. 2011. Negative Binomial Regression, $2^{\text {nd }}$ Edition. New York: Cambridge University Press.

Huber, John. 1989. "Values and Partisanship in Left-right Orientations: Measuring Ideology". European Journal of Political Research 17(5): 599-621. 
Imai, Kosuke, Gary King y Olivia Lau. 2006. "Zelig: Everyone's Statistical Software". Recuperado el 10 de mayo de 2010 en http:/ /GKing.Harvard.Edu/zelig.

Indridason, Indridi. 2008. "When to Run and When to Hide: Electoral Coordination and Exit". Economics \& Politics 20(1): 80-105.

Johnston, Richard y Fred Cutler. 2009. "Canada: The Puzzle of Local Three-Party Competition". En Duverger's Law of Plurality Voting. The Logic of Party Competition in Canada, India, the United Kingdom and the United States, editado por Bernard Grofman, André Blais y Shaun Bowler. New York: Springer, 83-96.

Jones, Mark. 1995. Electoral Laws and the Survival of Presidential Democracies. Notre Dame: University of Notre Dame Press.

Jones, Mark. 1999. "Electoral Laws and the Effective Number of Candidates in Presidential Elections". The Journal of Politics 61(1): 171-184.

Jones, Mark. 2004. "Electoral Institutions, Social Cleavages, and Candidate Competition in Presidential Elections". Electoral Studies 23(1): 73-106.

King, Gary, Michael Tomz y Jason Wittenberg. 2000. "Making the Most of Statistical Analyses: Improving Interpretation and Presentation". American Journal of Political Science 44(2): 341-355.

Laakso, Markku y Rein Taagepera. 1979. "Effective Number of Parties: A Measure with Application to West Europe". Comparative Political Studies 12(1): 3-27.

Lago, Ignacio. 2004. "La coordinación electoral del nacionalismo gallego". Revista Internacional de Sociología 39: 35-61.

Lago, Ignacio y Ferrán Martínez i Coma. 2012. “Forgetting to Make Votes Count: The Role of Previous Democratic Experience". Electoral Studies 31(2): 413-421.

Mainwaring, Scott. 1993. "Presidentialism, Multipartism, and Democracy: The Difficult Combination". Comparative Political Studies 26(2): 198-228.

Mainwaring, Scott y Matthew Shugart. 1997. Presidentialism and Democracy in Latin America. New York: Cambridge University Press.

Myatt, David. 2007. "On the Theory of Strategic Voting". Review of Economic Studies 74(1): 255-281.

Myerson, Roger y Robert Weber. 1993. "A Theory of Voting Equilibria". American Political Science Review 87(1): 102-114.

Nohlen, Dieter. 2005a. Elections in the Americas. A Data Handbook Vol. 1 North America, Central America, and the Caribbean. New York: Oxford University Press.

Nohlen, Dieter. 2005b. Elections in the Americas. A Data Handbook Vol. 2 South America. New York: Oxford University Press.

North, Douglass. 1990. "A Transaction Cost Theory of Politics". Journal of Theoretical Politics 2(4): 355-367.

Osborne, Martin y Al Silivinsky. 1996. "A Model of Political Competition with Citizen-Candidates". The Quarterly Journal of Economics 111(1): 65-96.

Palfrey, Thomas. 1984. "Spatial Equilibrium With Entry". Review of Economic Studies 51(1): 139156.

Pedersen, Mogens. 1979. “The Dynamics of West European Party Systems: Changing Patterns of Electoral Volatility". European Journal of Political Research 7(1): 1-26.

Political Database of the Americas. S.f. Recuperado en http:/ / pdba.georgetown.edu/.

Prescott, Edward y Michael Vischer. 1977. "Sequential Location Among Firms with Foresight". Bell Journal of Economics 8: 378-393.

Rae, Douglas. 1971. The Political Consecuences of Electoral Laws. New Heaven: Yale University Press.

Reed, Steven. 1990. "Structure and Behaviour: Extending Duverger's Law to the Japanese Case". British Journal of Political Science 20(3): 335-356.

Reed, Steven. 2003. "What Mechanism Causes the M+1 Rule? A Simple Simulation". Japanese Journal of Political Science 4(1): 41-60. 
Sani, Giacomo y Giovanni Sartori. 1983. "Polarization, Fragmentation and Competition in Western Democracies". En Western European Party Systems: Continuity and Change, editado Hans Daadler y Peter Mair. London: Sage, 307-340.

Sartori, Giovanni. 1994. Ingeniería constitucional comparada. México, D.F.: Fundación de Cultura.

Schelling, Thomas. 1960. The Strategy of Conflict. Cambridge: Harvard University Press.

Shepsle, Kenneth y Ronald Cohen. 1990. "Multiparty Competition, Entry and Entry Deterrence in Spatial Models of Elections". En Advances in Spatial Theory of Voting, editado por James Enelow y Melvin Hinich. New York: Cambridge University Press, 12-45.

Shugart, Matthew y John Carey. 1992. Presidents and Assemblies: Constitutional Design and Electoral Dynamics. New York: Cambridge University Press.

Shvetzova, Olga. 1995. "Design of Political Institutions in Divided Societies". Ph.D. dissertation. California: California Institute of Technology.

Stokes, Susan. 2009. "Globalization and the Left in Latin America", manuscrito no publicado.

Taagepera, Rein y Matthew Shugart. 1989. Seats and Votes: The Effects and Determinants of Electoral Systems. New Heaven: Yale Univserity Press.

Tavits, Margit y Taavi Annus. 2006. “Learning to Make Votes Count: The Role of Democratic Experience". Electoral Studies 25(1): 72-90.

Taylor, Michael y Valentine Herman. 1971. "Party Systems and Government Stability". American Political Science Review 65(1): 28-37.

Weber, Sholmo. 1997. "Entry Deterrence in Electoral Spatial Competition". Social Choice and Welfare 15(1): 31-56.

Weingast, Barry y William Marshall. 1988. “The Industrial Organization of Congress; or, Why Legislatures, Like Firms, Are Not Organized Like Markets". The Journal of Political Economy 96(1): 132-163.

Diego Luján es Magíster en Ciencia Política por la Universidad de la República de Uruguay, y candidato a Doctor en Ciencia Política por la Universidad Nacional de San Martín (Argentina). Es docente e Investigador en el Departamento de Ciencia Política de la Facultad de Ciencias Sociales de la Universidad de la República, e investigador activo del Sistema Nacional de Investigadores de Uruguay. Correo electrónico: diego.lujan@cienciassociales.edu.uy 
- 